\title{
PAPER SURFACE EFFICIENCY AND INKJET COLOUR REPRODUCTION ON INVASIVE AND AGRO RESIDUE PAPERS
}

\author{
Igor Karlovits (D), Gregor Lavrič \\ Pulp and Paper Institute, Ljubljana, Slovenia
}

\begin{abstract}
Paper surface efficiency was developed by Preucil and was intended to define the colourimetry and geometrical part of the light reflection with paper surface values. The surface characteristics of paper, primarily its roughness and absorptivity affect colour reproduction beside the ink pigments or dye. Rougher paper surfaces scatter the light in a more non-uniform manner while paper absorption may cause ink vehicle absorption and thus decreasing gloss, or it may absorb both vehicles and pigment-producing an unexpected colour shift. The formula was upgraded with paper whiteness values as the basic formula did not explain the whole phenomena. These paper properties were the foundation of the paper standardisation for standardised printing. On the other hand, during inkjet printing, a limited amount of liquid is deposited onto a paper surface. When the droplet hits the surface, it starts to spread and wet the surface depending on the ink and paper properties and had different colourant deposition from the formulas used mainly for offset printing inks. In this paper, we have researched the modified surface efficiency formula applied to inkjet printing (HP PageWide Pro 477dw) on invasive and agro-residue papers with specific non-white paper shades. We have measured the dynamic liquid penetration using ultrasound measurement to model the surface behaviour of the inks and measured CIE LCH values of the samples and calculated the CIE $\triangle E_{00}$ colour differences and the influence of paper surface efficiency and paper shade influence on the colour reproduction. The results indicate that using recalculated Cobb Absorption values gives good negative linear correlation with the original formula regarding the magnitude of colour difference.
\end{abstract}

Keywords: paper surface efficiency, colour reproduction, invasive plant paper, agro-residue paper, inkjet

\section{INTRODUCTION}

In defining paper properties and their influence on colour, reproduction one has to take in care of different paper properties. An excellent overview of surface and optical properties of paper can be found in (Farnood, 2009). Several research papers (Jurič et al, 2013; Tutak, 2014) showed the influence of paper properties on the colour reproduction values and visual evaluation by observers. In inkjet printing like in all printing technologies, the measured and the observed colour is the result of ink/surface interaction, and as (Pauler et al, 2009) found that in-home and office inkjet printing Large colour gamut is governed by low ink penetration, a low light scattering of the substrate and small dot size. The non-ideal property of process inks and the continuous tone character of colour reproduction of the studied desktop inkjets explained the observed convex shape of the colour gamut. For plain paper, dye-based and pigment-based inks were shown to follow different mechanisms, with lower penetration of the pigment-based ink. Colour gamut could be increased by a surface treatment that further reduced the penetration, but this treatment worked only for the pigment-based inks. Internal sizing of plain paper had only a minimal influence on colour gamut for dye-based ink, even though the ink penetration was reduced. In their research (Parales et al, 2008) found that the colour gamut of papers printed in inkjet has an excellent linear correlation between lightness and gamut volume especially on glossy coated papers and indicate that the surface finish and grammage influence the colour gamut. Similar research was carried out by Lowell (2006) and Lundberg (2011). To test the influence of whiteness values of inkjet prints (Hu et al, 2017) tested papers with dyes without changing the surface properties. Their results indicate that the colourimetric print values (lightness and chroma values) increased with increasing paper whiteness up to a certain level, after which the paper whiteness did not have a noticeable impact on its print lightness and chroma values. The print colour ( $a^{*}$ and $b^{*}$ values) was affected by its corresponding paper colour. On the other hand in another study by (Fernandez-Reche et al, 2003) by testing 29 paper found no systematic correlation between colour reproduction and specific colourimetric properties of the types of paper: and proposed search for other physical (not just colourimetric) properties (for instance, gloss or ink absorption capacity). Many times, in inkjet printing, mainly coated papers are investigated due to better optical print quality, but also some research can be found regarding uncoated samples. For example, in their research ( $\mathrm{Li}$ et al, 2015) investigated the impact on ink penetration of the 
microstructure and hydrophilicity of the uncoated papers. Their experimental results indicated that paper specimens with the sizing agent were resistant to the ink, resulting in slow and shallow ink penetration. Paper containing filler had a more hydrophilic surface and porous structure, leading to faster and deeper ink penetration. However, the calendering operation could make the paper structure more compact and reduce the porosity and penetration depth. When an appropriate combination of sizing agent, filler content, and the calendering process was utilised, a more stable hue could be produced with improvements in optical density, saturation, and colour.

All these researches regarding the interaction of inks, paper surfaces were an underlying foundation in the development of printing standards like the ISO 12647-2. The "standardised" paper types were developed by the PaperDam Group which has used the so-called surface index two which was based on PPS roughness and gloss in correlation with whiteness to specify the paper types used in print standardisation (Martilla et al, 2011). These metrics provided a good foundation for white and near white papers, but what about non-white papers with very distinctive paper shades, where no bleaching, coating pigments or optical brighteners are used and are not part of the standardised paper groups? How to evaluate them with a standard metric which includes the paper properties and colour values? Invasive plant papers and agro-residue papers are papers which utilise fibres from different sources (agro residues, dedicated crops or industrial waste). They provide alternative fibre sourcing beside the standard wood pulp solutions. Due to their specific fibres which are not bleached, and no extra whitening is used to retain as much as possible the sustainable and circular nature of these paper. Due to these properties, they need another approach regarding investigating their influence on their colour reproduction.

One of the solutions is to use the paper surface efficiency formula. During the first phase of the colour reproduction control development, Preucil (1963) proposed the paper surface efficiency formula (PSE), which defines the colour reproduction values after ink-paper interaction. The formula includes (geometrical reflection - gloss) and ink absorbency in (\%) but no other optical property is included, and this formula was based on densitometric measurements which were used at that time:

$\mathrm{PSE}=(100-\mathrm{A})+\mathrm{G} / 2$

Where PSE - is paper surface efficiency in \%

A- is ink absorbency of paper in \%

$\mathrm{G}$ - is paper gloss measured with $75^{\circ}$ angle gloss meter

This formula was also tested by (Charoenpholphibool, 1989) using offset inks on coated and uncoated papers. The densitometric values were changed for colourimetric, and results indicate that as the PSE increased, hue error and greyness decreased. The research found that the correlations were significant and were used to predict the colourimetric responses $\left(C^{*}, L^{*}\right)$ of ink when printed on papers having different PSE.

In another study by (Jeng-Rung Ho, 1991) the formula was extended by surface roughness measurements and was tested for predicting solid inking and printing gloss of offset printing. From the correlation matrix analysis, the new PSE equation yielded a higher correlation coefficient than the old PSE equation to predict better printed SID and print gloss. The formula they propose is:

NEW PSE $=0.6 / \mathrm{R} \times 100 \%+\mathrm{G} / 2$

Where $\mathrm{R}$ is the Print Parker Surface Roughness in $\mu \mathrm{m}$

As they have omitted the absorption factor to surface roughness factor, this would be more useful for offset printing where the pigment is on the paper surface, while in inkjet the dye goes into the paper surface, so this formula should be tested regarding different printing techniques.

In their paper (Yang et al, 2013) based on the research by (Yang et al, 2010) proposed a modified formula where they have included the whiteness factor (and weighted gloss to a lower number):

PSE $($ modified $)=100+0.8 \mathrm{G}+0.2 \mathrm{~W}-\mathrm{A} / 2$

Where the $\mathrm{W}$ - is the paper whiteness measurement in \%

The new formula was tested with laboratory offset test and using the Preucil procedure (KN Ink Absorbency). The results indicate the modified PSE formula can be used to predict ink colour effect effectively with hue error of magenta ink and greyscale of cyan ink on the paper, which provides data reference for paper make-ready, colour correction, and printing quality control. These results should help when lacking paper during printing to proceed with a paper substitute, which has close PSE values, rather than whiteness or another optical property. For example, in ISO 12647-2:2017 for offset printing (for 
other printing technologies no specific paper parameters are applied) pure optical properties (paper whiteness, gloss, paper CIE L*a*b* and fluorescence) are defined but no absorption value. Absorption value influences the optical density, CIE Lab and gloss values due to ink levelling and ink vehicle and pigment separation processes. So, this parameter is more or less »hidden « from the printers as paper mills can change formulations to fulfil the optical requirements. However, a slight change in pigment coating orientation (more precisely the imbibition process of ink vehicle draining in the porous structure) can influence the speed and ratio of absorbency and thus influence the optical properties. The paper mills traditionally check the paper absorption more often with the Cobb method, which is a static approach and expresses information about the water absorption capacity of paper samples. Based on this information, the sizing ratio is determined. The KN ink staining test can be used (just like in the original Preucil method), but some researchers did not find a high correlation between KN ink test and real ink behaviour on the press. This variation is due to the different nature of some $\mathrm{KN}$ or other staining inks used in paper and printing research. In their paper (Cigula et al, 2019) emphasise additional paper properties which are not in basic defined paper properties like smoothness and surface free energy which also have influencing factor on the ink - paper interaction and finally on the colourimetric values.

Our research aim was to try to modify and test the surface efficiency formula for inkjet printing and test the applicability on invasive and agro-residue papers which have brown shades and cannot be classified in "standardised" paper types. The modification involves the measurement of the absorption by using ultrasound methods previously also used by (Sharma, 2005) which found a strong correlation of ultrasound response with inkjet print quality parameters, dot area and print density, for photographic quality papers.

\section{METHODS AND MATERIALS}

All the papers were produced at the Pulp and Paper Institute in Ljubljana, Slovenia on a pilot paper machine. The traditional softwood and hardwood fibres were combined with different alternative fibres. The alternative fibres were from invasive plant's species obtained in urban harvesting and from planned to harvest plants miscanthus and tomato stems as agro-waste. At the same time, the jute bags from coffee transport were upcycled as a fibre source for papermaking. All the alternative (invasive and agrowaste fibres) were delignified and processed for the appropriate papermaking. Cationic starch, surface sizing and some fillers were added to gain basic printability properties. The primary material properties of the tested papers are presented in Table 1.

Table 1: The primary material properties of the invasive and agro-based papers

\begin{tabular}{|c|c|c|c|c|c|c|}
\hline Parameter & Jute & Miscanthus & $\begin{array}{c}\text { Canadian } \\
\text { Goldenrod }\end{array}$ & $\begin{array}{c}\text { Black } \\
\text { Locust }\end{array}$ & $\begin{array}{c}\text { Tomato } \\
\text { stem }\end{array}$ & $\begin{array}{c}\text { Japanese } \\
\text { Knotweed }\end{array}$ \\
\hline Grammage (g/m²) & 85 & 125 & 105 & 120 & 115 & 110 \\
\hline Alternative fibers (\%) & 35 & 67 & 45 & 40 & 20 & 40 \\
\hline Cationic starch (\%) & 1 & 0,75 & 1 & 1 & 1 & 1 \\
\hline Fillers (\%) & 10 & 0 & 10 & 10 & 5 & 10 \\
\hline Surface sizing (\%) & 3 & 2 & 3,5 & 3,5 & 4 & 3 \\
\hline
\end{tabular}

The prints were made by HP PageWide Pro $477 \mathrm{dw}$ inkjet printer which uses dye-based inks. All the paper samples were printed with a cyan colour patch in $100 \%$ ink value. The colourimetry values of the printed patches were measured following ISO 13655:2017 standard by using i1Pro2 spectrophotometer, which uses directional geometry and D50/2 ${ }^{\circ}$ setup using standard white backing. The paper values (CIE Lab and D65 whiteness) were measured using Datacolor Elrepho spherical spectrophotometer using d/0 geometry using $\mathrm{D} 65 / 10^{\circ}$ values. To define the absorption rate of the paper and the ink penetration, we have used additional measurements, as the liquid penetration dynamics, for what ultrasound measurements with Emtec PDA C.02 measuring device was applied. 16\% isopropanol mixture was the working fluid to define and simulate dye penetration ratio into the substrate. The original and modified surface efficiency formula uses Absorption value in \%, obtained from a staining test. This type of the oil/varnish component absorption rate cannot be used for inkjet printing due to different ink/dye composition and ink adhesion behaviour. For ultrasound measurements which are signal intensity/time values, we have chosen the t95 parameter and the A30 (internally recalculated Cobb water absorption value) which defines when the 
intensity drops to $95 \%$ of its original values. The paper gloss was measured using the $75^{\circ}$ angles with Lehman gloss meter.

\section{RESULTS}

The results of the samples basic properties needed for the calculation are presented in Table 2 . The values for the surface efficiency were calculated according to the original formula provided by Preucil, modified with 2 absorption values. In formulas, we have changed the A \% value to t95 value as both are dimensionless and multiplicated by 100 because of the small number for more straightforward calculation, and used calculated A30 value which is the approximation of the Cobb value. The resulting curves from the absorption measurement are presented in Figure 1. The results indicate differences in the critical region of the first few second when the inkjet inks are absorbed.

Table 2: Paper properties and surface efficiency calculation

\begin{tabular}{|c|c|c|c|c|c|c|c|}
\hline Paper sample & $\begin{array}{c}\text { W D65 } \\
(\%)\end{array}$ & Gloss (\%) & $\begin{array}{c}\text { A- } \\
\text { t95\% }\end{array}$ & A30 & $\begin{array}{c}\text { PSE 1-t95 } \\
(\%)\end{array}$ & $\begin{array}{c}\text { PSE 1- } \\
\text { A30* }\end{array}$ & $\begin{array}{l}\text { PSE 2** } \\
(\%)\end{array}$ \\
\hline Jute & 52,58 & $5,48 \pm 0,39$ & 35,6 & 27,8 & 29,46 & 33,36 & 43,55 \\
\hline Miscanthus & 31,13 & $3,6 \pm 0,31$ & 18,6 & 26,5 & 38,9 & 34,95 & 41,32 \\
\hline $\begin{array}{c}\text { Canadian } \\
\text { Goldenrod }\end{array}$ & 37,90 & $6,01 \pm 0,57$ & 15,5 & 25,1 & 39,24 & 34,45 & 43,64 \\
\hline Black Locust & 27,56 & $2,88 \pm 0,1$ & 13,3 & 22,1 & 41,91 & 37,51 & 42,86 \\
\hline Tomato stem & 43,17 & $4,1 \pm 0,52$ & 5,8 & 27,8 & 45,05 & 34,05 & 42,05 \\
\hline $\begin{array}{c}\text { Japanese } \\
\text { Knotweed }\end{array}$ & 31,08 & $3,03 \pm 0,08$ & 5,4 & 24,4 & 45,78 & 36,3 & 42,11 \\
\hline
\end{tabular}

*Original Preucil formula modified by us (KN absorption - changed to A30 from PDA measurements)

**modified formula based on the work by (Yang et al, 2010)

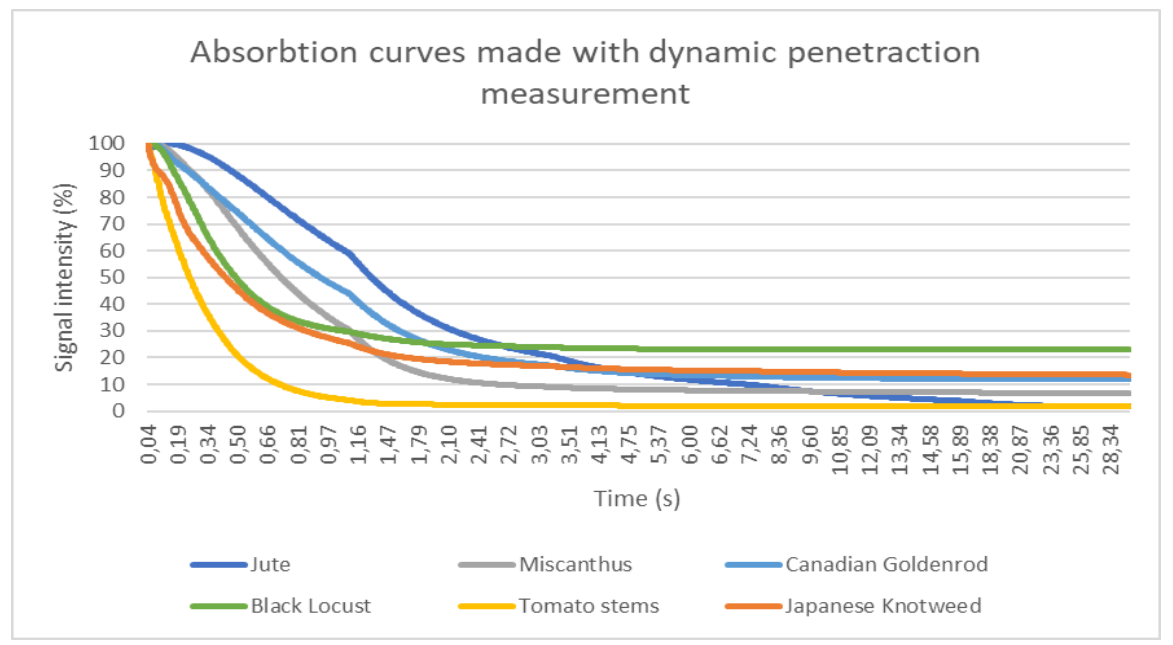

Figure 1: Absorption curves of different papers

From Figure 1. we can observe that the quickest absorption in the first few seconds had the Tomato stem, Japanese knotweed and Black Locust paper while the paper made from jute bags, Canadian Goldenrod and Miscanthus had lower absorption in the first 2-3 seconds. After the 3 seconds, more or less, all paper had similar absorption curves. The changes in the first 2-3 seconds, which can be differentiated by the t95 value had no value for the paper surface efficiency value but the whole curve calculation during the $30 \mathrm{~s}$ period. These results indicate that the overall absorption is more important than the first contact effects in the calculation of colour reproduction and colourimetric changes. The colourimetric changes of printed ink (100\% cyan) patch are presented in Table 3. 
Table 3: Colourimetric values of the $100 \%$ Cyan patch (CIE Lab of ideal cyan was taken as $L=57, a=-40, b=-52$ )

\begin{tabular}{|c|c|c|c|c|}
\hline Samples & Cyan D & CIE Lab & CH & $\Delta \mathrm{E} 00$ \\
\hline Jute & 0.82 & $56,29 /-16,50 /-23,87$ & $29,02 / 235,34$ & 12,24 \\
\hline Miscanthus & 0.86 & $51,91 /-17,29 /-10,73$ & $20,35 / 211,79$ & 18,12 \\
\hline $\begin{array}{c}\text { Canadian } \\
\text { Goldenrod }\end{array}$ & 0.86 & $53,54 /-20,23 /-11,81$ & $23,43 / 210,26$ & 16,82 \\
\hline Black Locust & 0.88 & $49,86 /-13,66 /-10,06$ & $16,97 / 216,34$ & 19,41 \\
\hline Tomato stem & 0.85 & $54,41 /-17,49 /-18,65$ & $25,58 / 226,80$ & 13,83 \\
\hline $\begin{array}{c}\text { Japanese } \\
\text { Knotweed }\end{array}$ & 0.86 & $51,28 /-14,44 /-11,91$ & $18,72 / 219,51$ & 18,05 \\
\hline
\end{tabular}

From Table 2., it is evident that the colour difference is very high (in comparison with "ideal" cyan ink taken from widest available CMYK gamut) as we used papers with distinctive shades and inkjet printing which is absorbent especially in papers which are not coated.

To see the usefulness of our modified Absorption value in the two paper surface efficiency formulas we calculated both $\mathrm{t} 95$ and $\mathrm{A} 30$ factor with $\Delta \mathrm{E}_{00}$ values as a reference for colour quality reproduction, where the $A 30$ value produced a better coefficient of determination $R^{2}$ for linear correlation 0,75 ( $r$ Pearson coefficient of 0,86 ); (the t95 factor had only $r=0,28$ ). So here the surface efficiency value is negatively linear to good print quality (lower PSE values gives smaller colour difference values from the referent colour). The low PSE value comes from high gloss and high A30 value.

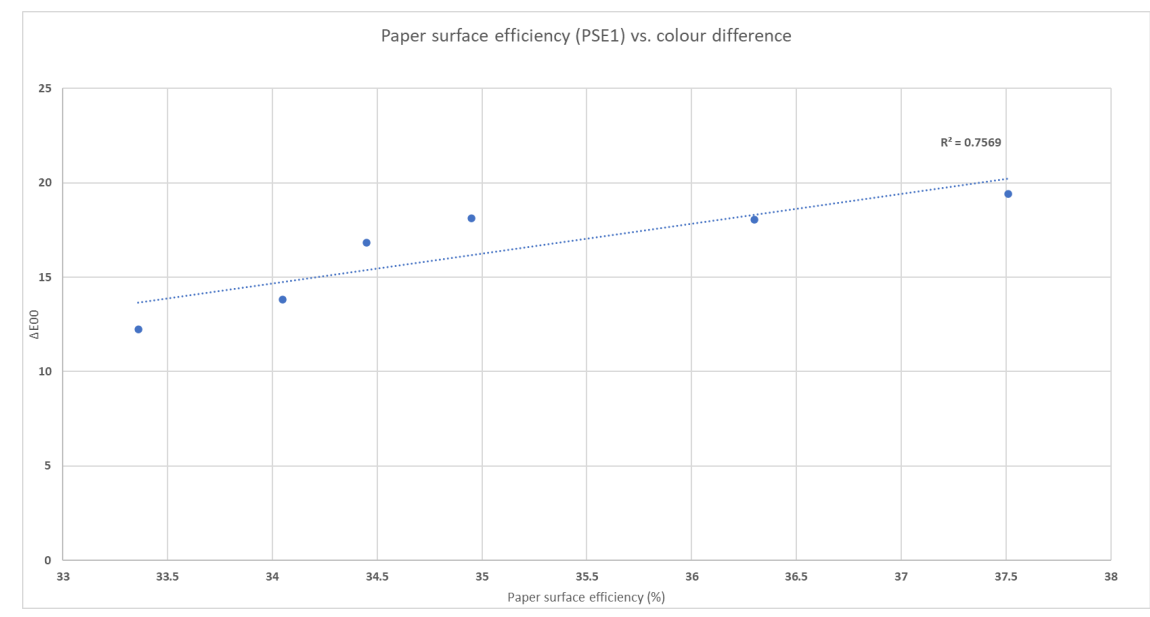

Figure 2: Correlation between Paper surface efficiency PSE1 and colour difference $\left(\Delta E_{00}\right)$

When comparing the two-surface efficiency formula with the changed absorption values, the second one which uses coefficients for gloss and whiteness did not show the correlation between colour difference. The calculated values indicate that the papers used in this study with very low whiteness cannot use the whiteness modified formula without further adjustments for water-based inkjet prints.

\section{CONCLUSION}

Any printer which confronts printability problems regarding achieving standardised values has to rely on the required optical properties and the »black box « knowledge of the paper mills that they will find a solution for achieving the desired colourimetric values. On the other hand, with agro-residue or invasive plant papers which are uncoated and do not contain optical brighteners, the usual paper properties used for print evaluation based on whiteness cannot be used. The whiteness formulas were developed for the characterisation of white and blue shades of white papers. For paper which are uncoated and non-white papers, the surface efficiency formula can be used to predict print quality. To use it for inkjet printing, the change of absorption component is needed (originally developed for $\mathrm{KN}$ inks which mimic the offset 
printing) to digitally calculated A30 absorption values. The changed formula provided excellent linear correlation with $r=0,86$, when used with the original Preucil formula developed in the 60 s of the previous century. The results show that with these measurements, we can "predict" the size of colour difference from ideal ink. As these papers have very distinctive shades, future research will include besides paper whiteness values also paper shade values in the calculation, as the human observer is differently sensitive to lightness, chroma and hue. The use of modified paper surface efficiency formula (adjusted for inkjet printing) can be beneficial in future colourimetric measurement research due to non -existent standardisation of these kinds of papers. Also, the results can be useful for paper production to optimise the alternative fibre content of papers as these are more variable than in large scale wood pulp production.

\section{ACKNOWLEDGEMENTS}

We want to thank Tea Kapun and the project APPLAUSE "From harmful to useful with citizen's led activities", co-financed by the European Union under the European Regional Development Fund, through the initiative Urban Innovative Actions, for providing alien plant species papers.

\section{REFERENCES}

[1] Charoenpholphibool, W.: "The Study of paper surface efficiency", MSc thesis, Rochester Institute of Technology, New York, 1989.

[2] Cigula, T., Tomašegović, T., Hudika, T.: "Effect of the paper surface properties on the ink transfer parameters in offset printing", Nordic Pulp \& Paper Research Journal 34(4), 540-549, 2019. doi: 10.1515/npprj-2019-0018.

[3] Farnood, R.: "Review: Optical properties of paper: theory and practice", (In Advances in Pulp and Paper Research, Oxford 2009), pages 273-352.

[4] Hu, G., Fu, S., Chu, F., Lin, M.: "Relationship between Paper Whiteness and Color Reproduction in Inkjet Printing”, Bio Resources 12(3), 4854-4866, 2017. doi: 10.15376/biores.12.3.4854-4866.

[5] Jurič, I., Karlović, I., Tomić, I., Novaković, D.: “Optical paper properties and their influence on colour reproduction and perceived print quality", Nordic Pulp \& Paper Research Journal 28(2), 264-273, 2013.

[6] Li, R., Zhang, Y., Cao, Y., Liu, Z.: "Ink Penetration of Uncoated Inkjet Paper and Impact on Printing Quality", Bio Resources 10(4), 8135-8147, 2015.

[7] Lowell, V.: "Ink-Jet Inks and Substrates - Novel Approaches for Their Physical and Optical Properties Characterisation", Graduate thesis, Western Michigan University, Michigan, 2006.

[8] Lundberg, A.: "Ink-paper interactions and effect on print quality in inkjet printing", MSc thesis, Mid Sweden University, Sudsvall, 2011.

[9] Martilla, J., Carl, G., Lanat, L.: "Standardisation Update and Paperdam Group", PPA's Paper Profile working group, 2011

URL: http://www.paperdam.org/cms/upload/P_PPA_London_2011/JM_Paperdam_status_126472_2011_02.pdf> (last request 2020-09-03.).

[10] Pauler, N., Norberg, O., Edström, P.: "Mechanism involved in the optical interaction between the ink and substrate for Home \& Office inkjet printing", Proceedings of $36^{\text {th }}$ International Research Conference of larigai 2009, (IARIGAI, Stockholm, Sweden, 2009), pages 372-382.

[11] Preucil, A.: "New Method of Rating the Efficiency of Paper for Color Reproduction", GATF Research Progress General, 1963.

[12] Sharma, N.: "Use of ultrasound to predict inkjet print quality", MSc thesis, Miami University, Oxford, Ohio, 2005.

[13] Tutak, D.: "Comparing the Color Gamuts Of Different Paperboard Surfaces Used in Package Printing", AJIT-e: Online Academic Journal of Information Technology 5(17), 57-66, 2014. doi: 10.5824/1309-1581.2014.4.004.x.

[14] Yang, Y., Hou, Q., Cao, Z., Liu, Q.: "Research on the Revision and Application of the Paper Surface Efficiency Formula", Advanced Materials Research 156-157, 868-872, 2010. doi: 10.4028/www.scientific.net/AMR.156-157.868.

[15] Yang, Y., Gao, Q., Liu, Q.: "The Influences of New Paper Surface Efficiency on Printing Quality", Applied Mechanics and Materials 477-478, 374-378, 2013. doi:

10.4028/www.scientific.net/AMM.477-478.374. 


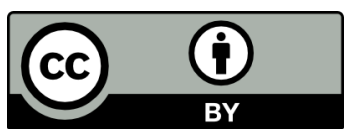

(C) 2020 Authors. Published by the University of Novi Sad, Faculty of Technical Sciences, Department of Graphic Engineering and Design. This article is an open access article distributed under the terms and conditions of the Creative Commons Attribution license 3.0 Serbia

(http://creativecommons.org/licenses/by/3.0/rs/). 